\title{
Unraveling Charge Transfer in CoFe Prussian Blue Modified $\mathrm{BiVO}_{4}$ Photoanodes
}

Benjamin Moss, ${ }^{\dagger, \#}$ Franziska Simone Hegner, ${ }^{\ddagger}, \#$ Sacha Corby, ${ }^{\dagger}$ Shababa Selim, ${ }^{\dagger}$ Laia Francàs, ${ }^{*}, \dagger$

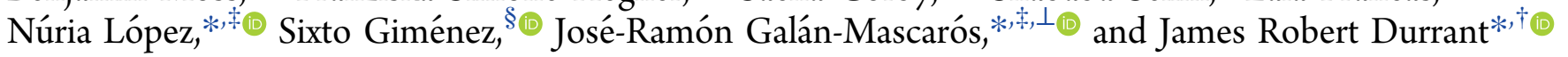

${ }^{\dagger}$ Department of Chemistry, Imperial College London, Imperial College Road, London SW7 2AZ, United Kingdom

${ }^{\ddagger}$ Institut Català d'Investigació Química (ICIQ), The Barcelona Institute of Science and Technology (BIST), Avinguda Països Catalans 16, 43007 Tarragona, Spain

${ }^{\S}$ Institute of Advanced Materials (INAM), Universitat Jaume I, 12006 Castelló, Spain

${ }^{\perp}$ ICREA, Passeig de Lluís Companys 23, 08010 Barcelona, Spain

Supporting Information

ABSTRACT: Catalyst modification of metal oxide photoanodes can result in markedly improved water oxidation efficiency. However, the reasons for improvement are often subtle and controversial. Upon depositing a CoFe Prussian blue (CoFe-PB) water oxidation catalyst on $\mathrm{BiVO}_{4}$, a large photocurrent increase and onset potential shift (up to $0.8 \mathrm{~V}$ ) are observed, resulting in a substantially more efficient system with high stability. To elucidate the origin of this enhancement, we used time-resolved spectroscopies to compare the dynamics of photogenerated holes in modified and unmodified $\mathrm{BiVO}_{4}$ films. Even in the absence of strong positive bias, a fast (pre-ms), largely irreversible hole transfer from $\mathrm{BiVO}_{4}$ to $\mathrm{CoFe}-\mathrm{PB}$ is observed. This process retards recombination, enabling holes to accumulate in the catalyst. Holes in CoFe-PB remain reactive, oxidizing water at a similar rate to holes in pristine $\mathrm{BiVO}_{4}$. CoFe-PB therefore enhances performance by presenting a favorable interface for efficient hole transfer, combined with the catalytic function necessary to drive water oxidation.

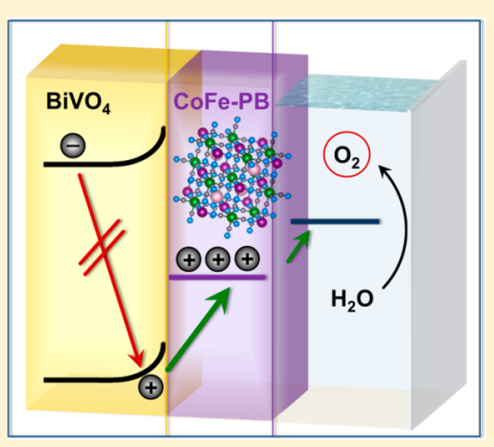

$\mathrm{M}$ etal oxides, such as $\mathrm{TiO}_{2},{ }^{1} \mathrm{Fe}_{2} \mathrm{O}_{3}{ }^{2} \mathrm{WO}_{3}{ }^{3}$ and $\mathrm{BiVO}_{4}{ }^{4}$ have been extensively studied as candidate photoanodes for water oxidation (WO). Despite their ideally aligned valence bands, which provide a large driving force for $\mathrm{WO}^{5}$ large additional positive applied potentials are still required to access high photon-to-current conversion efficiencies. A common approach to reduce the need for such strong positive potentials is to deposit an electrocatalyst on the surface of the photoanode to act as a cocatalyst. This strategy is often successful, yielding enhanced photocurrents and reduced WO onset potentials. ${ }^{4,6-11}$ However, improvements in performance are not necessarily linked to improved WO kinetics. Other mechanisms, such as retarding electron-hole recombination within the semiconductor, may dominate. For example, deposition of cobalt phosphate $\left(\mathrm{CoP}_{\mathrm{i}}\right)^{12}$ on dense $\mathrm{BiVO}_{4}$ leads to a $100-250 \mathrm{mV}$ negative shift in the onset potential of WO. ${ }^{10}$ Independent studies of $\mathrm{CoP}_{\mathrm{i}}$ modified films using electrochemical impedance spectroscopy, intensity modulated photocurrent spectroscopy, ${ }^{13}$ and transient absorption spectroscopy $(\mathrm{TAS})^{10}$ indicate that this improvement is not related to hole transfer to $\mathrm{CoP}_{\mathrm{i}}$ (i.e., charge separation) and/or WO catalyzed by $\mathrm{CoP}_{\mathrm{i}}$ but rather stems from retarded surface recombination within $\mathrm{BiVO}_{4}$. Intriguingly, unfavorable interfacial kinetics for hole transfer to $\mathrm{CoP}_{\mathrm{i}}$ are observed, despite this transfer having a large driving force, ${ }^{14}$ with time scales 210 times slower than direct $\mathrm{WO}$ on the $\mathrm{BiVO}_{4}$ surface (which has a characteristic time constant of ca. $0.5 \mathrm{~s}) .^{14-16}$ Although some oxidation of $\mathrm{CoP}_{\mathrm{i}}$ was observed under WO conditions, this oxidation of $\mathrm{CoP}_{\mathrm{i}}$ did not contribute significantly to the overall WO flux ${ }^{14}$ due to $\mathrm{CoP}_{\mathrm{i}}$ 's relatively slow WO kinetics (on the order of $10^{-3} \mathrm{~s}^{-1}$ per active site). ${ }^{17}$ As such, finding a co-catalyst that acts by directly enhancing WO on its active sites remains a key challenge for the development of more efficient photoanodes. While studies have reported indirect evidence for such performance enhancement, ${ }^{18}$ we focus herein on a direct kinetic study of a promising candidate system, cobalt iron Prussian blue on $\mathrm{BiVO}_{4}$.

Cobalt hexacyanoferrate, the cobalt-iron analogue of Prussian blue (CoFe-PB), is a robust, effective, and inexpensive electrocatalyst. CoFe-PB shows low WO onset potentials and exceptional stability, even in acidic media, while being simple and cost-effective to prepare. ${ }^{19-22}$ Critically, CoFe-PB exhibits much faster WO kinetics than $\mathrm{CoP}_{\mathrm{i}}\left(1.4 \mathrm{~s}^{-1}\right.$ for $\mathrm{CoFe}-\mathrm{PB}$ vs 2 $\times 10^{-3} \mathrm{~s}^{-1}$ for $\mathrm{CoP}_{\mathrm{i}}$ per active site). ${ }^{17,19}$ It has recently been demonstrated that CoFe-PB modification of mesostructured

Received: November 17, 2018

Accepted: December 26, 2018

Published: December 26, 2018 

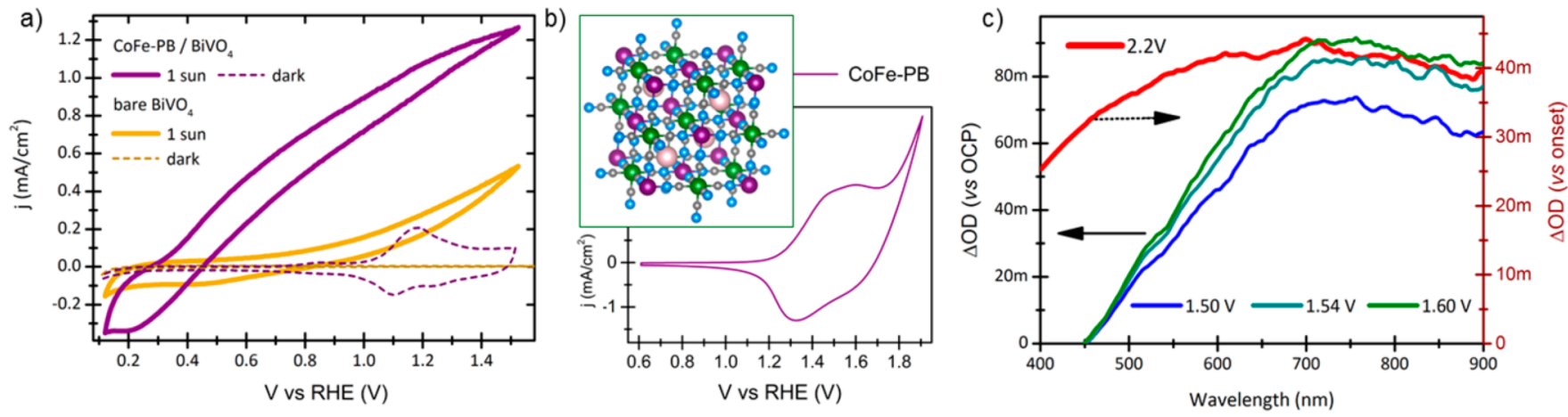

Figure 1. Cyclic voltammograms (a) of bare $\mathrm{BiVO}_{4}$ (yellow) and $\mathrm{CoFe}-\mathrm{PB}$ modified $\mathrm{BiVO}_{4}$ (purple) with and without illumination (1 sun) and (b) of CoFe-PB alone (in the dark), recorded at $50 \mathrm{mV} / \mathrm{s}$ at $\mathrm{pH} 7\left(0.1 \mathrm{M} \mathrm{KP}_{\mathrm{i}}\right.$ buffer). Inset: Ideal (fcc) crystal structure of CoFe-PB with octahedrally coordinated Co (purple) and Fe (green) centers. Differential absorbance spectra (c) observed upon the oxidation of CoFe-PB/ FTO. When potentials positive of the open-circuit potential are applied (green/blue lines, referenced against OCP), an asymmetric peak with a broad maximum at around $700-800 \mathrm{~nm}$ is observed. At potentials exceeding the onset potential of WO electrocatalysis (red line, referenced against the onset potential), a broader spectrum is observed with a maximum between 600 and $700 \mathrm{~nm}$.

$\mathrm{BiVO}_{4}$ photoanodes produces substantial improvements in both the onset potential and photocurrent, maintaining stability for over $50 \mathrm{~h}^{18}$ Preliminary investigations of CoFe$\mathrm{PB}$ modified $\mathrm{BiVO}_{4}$, using photoelectrochemical impedance and hole scavenger studies, suggest that the origin of this improvement is distinct from the effect of $\mathrm{CoP}_{\mathrm{i}}$ (i.e., suppression of surface recombination within $\mathrm{BiVO}_{4}$ ) and related to more efficient hole transfer to water, presumably via CoFe-PB. ${ }^{18}$ These findings are consistent with hybrid density functional theory calculations, which predict the existence of a strong energetic offset (thermodynamic driving force) for hole transfer between the valence band of $\mathrm{BiVO}_{4}$ and $\mathrm{CoFe}-\mathrm{PB}{ }^{18,23}$

Herein, we present the first study of the effect of CoFe-PB modification of $\mathrm{BiVO}_{4}$ electrodes on the kinetics of photogenerated holes. Using time-resolved absorption spectroscopies, we demonstrate that $\mathrm{BiVO}_{4}$ holes quickly and efficiently transfer to $\mathrm{CoFe}-\mathrm{PB}$, leading to persistent oxidized $\mathrm{CoFe}-\mathrm{PB}$ states even at very low bias. This capacity to separate charges at modest applied potential is coupled with effective catalytic functionality as accumulated holes in CoFe-PB form multiply oxidized states, which react with water with similar kinetics to unmodified $\mathrm{BiVO}_{4}$, despite a significant loss of oxidizing potential during charge separation.

Nanostructured $\mathrm{BiVO}_{4}$ photoanodes were fabricated by a previously described electrodeposition method and modified with CoFe-PB by sequential coating. ${ }^{18}$ Details of this synthesis and the film morphology (including SEM and TEM images of unmodified and $\mathrm{CoFe}-\mathrm{PB}$ modified $\mathrm{BiVO}_{4}$ ) can be found in the Supporting Information (Figure S1). CoFe-PB on FTO was prepared using a modified hydrothermal method, developed by Han et al. (see the SI for details). ${ }^{22}$ Consistent with previous reports, a thin $(<20 \mathrm{~nm})$ co-catalyst layer is observed on $\mathrm{BiVO}_{4}$ after sequential coating (Figure S1e,f). ${ }^{18}$ Due to the thinness of this layer, the ground state absorption spectra of CoFe-PB modified and unmodified $\mathrm{BiVO}_{4}$ films (Figure S2) do not significantly differ. We thus conclude that the enhancements in photocurrent are not caused by increased photon absorption, i.e., that photons are predominantly absorbed by $\mathrm{BiVO}_{4}$.

Cyclic voltammograms of CoFe-PB modified and unmodified $\mathrm{BiVO}_{4}$ in the dark and under simulated AM1.5 illumination are compared in Figure 1a. Negligible current was observed for unmodified $\mathrm{BiVO}_{4}$ in the dark. Upon CoFePB modification, a semireversible redox couple is observed in the dark at around $1.2 \mathrm{~V}_{\mathrm{RHE}}$, with electrocatalytic WO occurring after $1.6 \mathrm{~V}_{\mathrm{RHE}}$ (a value similar to that observed for CoFe-PB/FTO electrocatalysis). ${ }^{18}$ Taking the voltage required to attain $0.1 \mathrm{~mA} / \mathrm{cm}^{2}$ as the onset potential for photocurrent under 1 sun irradiation, a negative shift of $0.8 \mathrm{~V}$ of the onset potential is achieved upon CoFe-PB deposition (Figure S3), in agreement with previous reports. ${ }^{18}$ In order to confirm that the measured current is due to WO, gas chromatography experiments were carried out and a Faradaic efficiency close to $100 \%$ was achieved (Figure S4). ${ }^{18}$

Before considering the effect of CoFe-PB modification on the transient absorption of $\mathrm{BiVO}_{4}$, we first determined the effect of positive applied potential (oxidation) on the ground state absorption of CoFe-PB/FTO (employing a thicker, $\mathrm{CoFe}-\mathrm{PB}$ film to yield a measurable ground state absorption spectrum). When potentials positive of the open-circuit (resting) potential $\left(\mathrm{OCP}=1.1 \mathrm{~V}_{\mathrm{RHE}}\right)$ are applied, a broad and asymmetric (decaying more strongly toward shorter wavelengths) absorption difference spectrum is observed, peaking at around $700-800 \mathrm{~nm}$ (blue and green lines in Figure 1c; a full data set can be found in Figure S5). We attribute this to the oxidation of $\mathrm{CoFe}-\mathrm{PB}$ as it increases with applied potential and correlates strongly with the semireversible redox wave observed before the onset of catalytic current (Figures $1 \mathrm{~b}$ and S5c). CoFe-PB films are nonstoichiometric, likely containing a mixture of neighboring $\mathrm{Co}^{2+}$ and $\mathrm{Fe}^{2+}, \mathrm{Co}^{3+}$ and $\mathrm{Fe}^{2+}$ (and possibly $\mathrm{Co}^{2+}$ and $\mathrm{Fe}^{3+}$ ) centers, each with different coordination environments and redox potentials. ${ }^{24,25}$ Consequently, this redox wave cannot clearly be assigned uniquely to either a $\mathrm{Co}^{2+} / \mathrm{Co}^{3+}$ or a $\mathrm{Fe}^{2+} /$ $\mathrm{Fe}^{3+}$ couple. For simplicity, we hereafter refer to this oxidized state as "CoFe-PB" ${ }^{+26}$ At potentials positive of the electrocatalytic WO onset potential $\left(1.6 \mathrm{~V}_{\mathrm{RHE}}\right)$, a smaller, flatter difference spectrum with increased amplitude toward blue wavelengths is observed (red line in Figure 1c). This second spectrum exhibits a blue-shifted maximum compared to CoFe$\mathrm{PB}^{+}$. We assign this change in spectral shape to further oxidation of $\mathrm{CoFe}-\mathrm{PB}^{+}$, which again for simplicity we label "CoFe-PB ${ }^{2+»}$. The appearance of this second spectrum correlates with the onset of WO catalysis (Figure 1b.).

Utilizing the spectral fingerprints of oxidized CoFe-PB states to interpret our results, we now turn to TAS (see the SI for details), which we use to assess the effect of CoFe-PB modification on the kinetics of photogenerated holes in $\mathrm{BiVO}_{4}$. 
Figure 2a presents a comparison of the transient absorption spectra (at $10 \mathrm{~ms}$ ) of CoFe-PB modified and unmodified
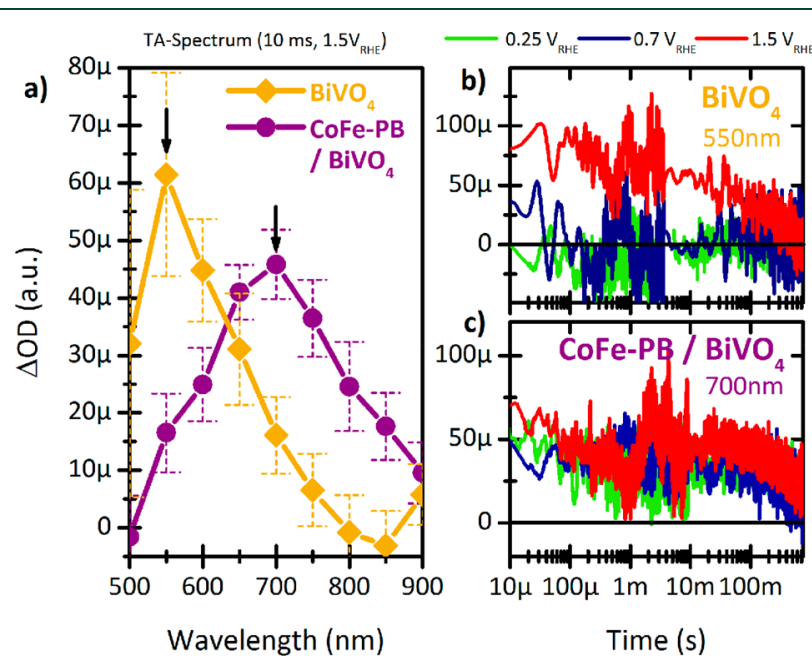

Figure 2. Comparison of the TA spectra of persistent photogenerated charges (recorded at $10 \mathrm{~ms}$ ) in CoFe-PB modified and unmodified $\mathrm{BiVO}_{4}(\mathrm{a})$. In unmodified $\mathrm{BiVO}_{4}$, a persistent transient related to surface holes is observed only at a strong positive potential ( $\left.1.5 \mathrm{~V}_{\mathrm{RHE}}\right)(\mathrm{b})$. Upon CoFe-PB modification, a persistent transient is observed at all potentials (even at $0.25 \mathrm{~V}_{\mathrm{RHE}}$ ) (c).

$\mathrm{BiVO}_{4}$ films, both at strong positive bias, while Figure $2 \mathrm{~b}, \mathrm{c}$ shows kinetics at selected wavelengths as a function of applied bias. Consistent with previous reports, ${ }^{16}$ unmodified $\mathrm{BiVO}_{4}$ exhibits a transient absorption spectrum peaking at $550 \mathrm{~nm}$ and decaying toward the near-IR. This spectrum has previously been assigned to $\mathrm{BiVO}_{4}$ surface holes. ${ }^{16}$ After $\mathrm{CoFe}-\mathrm{PB}$ modification, the amplitude at $550 \mathrm{~nm}$ is lost, and a new peak bearing a strong resemblance to the absorption of CoFe$\mathrm{PB}^{+}(\sim 700 \mathrm{~nm}$, decaying more strongly toward shorter wavelengths) is observed. Taken together, these results demonstrate efficient hole transfer from the $\mathrm{BiVO}_{4}$ surface to CoFe-PB.

An increase in the lifetime of photogenerated charges upon $\mathrm{CoFe}-\mathrm{PB}$ modification at low applied potentials is clearly visible in the TA kinetics of the electrodes (Figure $2 b, c$ ). Concordant with previous transient studies, strong positive bias $\left(1.5 \mathrm{~V}_{\mathrm{RHE}}\right)$ is required to produce persistent (with lifetime $\tau>10 \mathrm{~ms}$ ) photogenerated holes in unmodified $\mathrm{BiVO}_{4}$ (Figure 2b). ${ }^{16}$ However, after $\mathrm{CoFe}-\mathrm{PB}$ modification, persistent transient absorption is observed even at low applied potentials $\left(0.25 \mathrm{~V}_{\mathrm{RHE}}\right.$, Figure $2 \mathrm{c}$, see also spectra in Figure S6). Figure $2 \mathrm{c}$ also shows that the signal amplitude at early times $(10 \mu \mathrm{s})$ remains almost unchanged for tenths of $\mathrm{ms}$. This data indicates that hole transfer from $\mathrm{BiVO}_{4}$ to $\mathrm{CoFe}-\mathrm{PB}$ is largely complete by $10 \mu \mathrm{s}$. We attribute this increased hole lifetime to fast and efficient hole transfer. This is because hole transfer to CoFe-PB corresponds to a spatial separation of charge, which suppresses recombination even in the absence of a strong applied potential.

To better understand the fate of persistent separated holes in $\mathrm{CoFe}-\mathrm{PB}$ under operational conditions, we utilize photoinduced absorption spectroscopy (PIAS), employing a $5 \mathrm{~s}, 365$ nm LED pulse to simulate in operando conditions (details can be found in the SI and previous publications). ${ }^{14}$ Unmodified $\mathrm{BiVO}_{4}$ again shows a peak at $550 \mathrm{~nm}$, assigned to the accumulation of $\mathrm{BiVO}_{4}$ surface holes facilitated by a strong positive applied potential (Figure 3a). ${ }^{14-16}$ Upon CoFe-PB modification, the amplitude at $550 \mathrm{~nm}$ decreases and a new

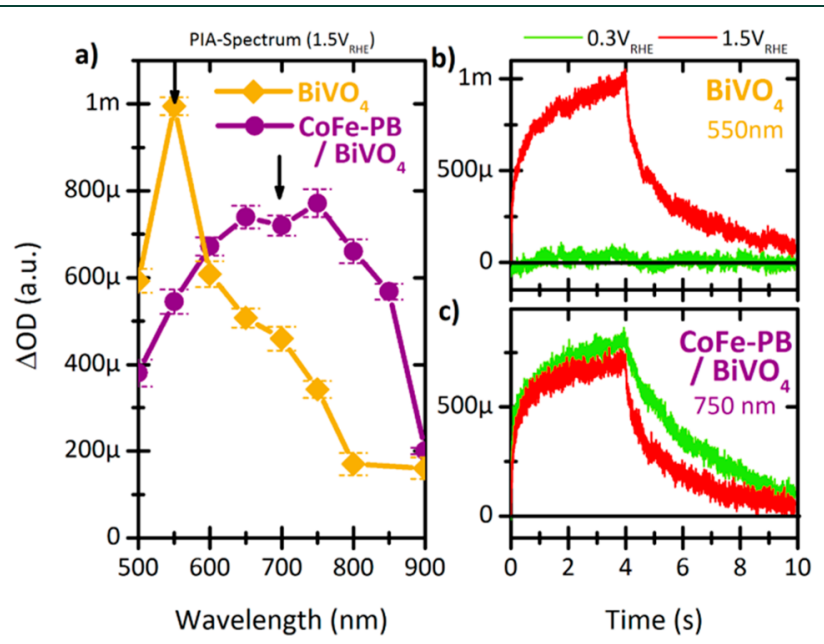

Figure 3. Comparison of the PIA spectra of CoFe-PB modified and unmodified $\mathrm{BiVO}_{4}$ films (a). In unmodified $\mathrm{BiVO}_{4}$, a strong positive potential $\left(1.5 \mathrm{~V}_{\mathrm{RHE}}\right)$ is required to observe a large growing transient, related to the accumulation of $\mathrm{BiVO}_{4}$ surface holes under illumination (b). Upon CoFe-PB modification, photogenerated charge accumulates even at potentials close to the onset potential of photocurrent, such as $0.3 \mathrm{~V}_{\mathrm{RHE}}(\mathrm{c})$.

peak consistent with oxidized CoFe-PB $(\sim 650-750 \mathrm{~nm}$; see the following section for discussion of spectral shape and CoFe-PB oxidation state) is observed (Figure 3a). This change is broadly similar to the spectral change observed in TAS upon $\mathrm{CoFe}-\mathrm{PB}$ modification, further supporting the conclusion that photogenerated holes transfer to, and accumulate in, CoFe-PB rather than $\mathrm{BiVO}_{4}$.

The effect of the CoFe-PB layer on the dynamics of accumulated charges can be found by examining the PIA kinetics as a function of applied bias (Figure $3 \mathrm{~b}, \mathrm{c}$ ). At lower applied potentials, a negligible PIA signal is observed for unmodified $\mathrm{BiVO}_{4}$, indicating that only a few holes accumulate (Figure $3 \mathrm{~b}$ ). Under large positive bias, a strong PIA signal is observed, indicative of the accumulation of surface holes under in operando conditions, consistent with our TAS studies (Figure 2b). After CoFe-PB modification, such strong positive potentials are no longer required to observe large PIA signals (Figure 3c), again consistent with our TAS data (Figure 3c). The decay kinetics of the PIA traces at high anodic bias (where recombination processes are strongly suppressed for both electrodes) gives a direct indication of the WO kinetics under these in operando conditions. ${ }^{14}$ In unmodified $\mathrm{BiVO}_{4}$, an initial rate analysis yields a WO time constant from $\mathrm{BiVO}_{4}$ surface holes on the order of seconds (Figures $3 \mathrm{~b}$ and S8a), consistent with our previous reports. ${ }^{15,16}$

The PIA signal decays for CoFe-PB modified $\mathrm{BiVO}_{4}$, assigned to oxidized $\mathrm{CoFe}-\mathrm{PB}$ states, exhibit broadly similar decay kinetics to $\mathrm{BiVO}_{4}$ holes, indicative of similar WO oxidation kinetics on both $\mathrm{BiVO}_{4}$ and CoFe-PB (Figure 3c). However, in contrast to $\mathrm{BiVO}_{4}$, significant PIA signal is observed for CoFe-PB modified $\mathrm{BiVO}_{4}$ even at $0.3 \mathrm{~V}_{\mathrm{RHE}}$, with the PIA decay kinetics accelerating with increasing applied potential. The signal decaying faster cannot be attributed to recombination being suppressed as this would result in retardation of this decay. This acceleration with increased positive bias coincides with a blue shift of the PIA spectrum 
(Figure 4a). This is analogous to the blue shift observed with increased positive bias in our spectroelectrochemical data for

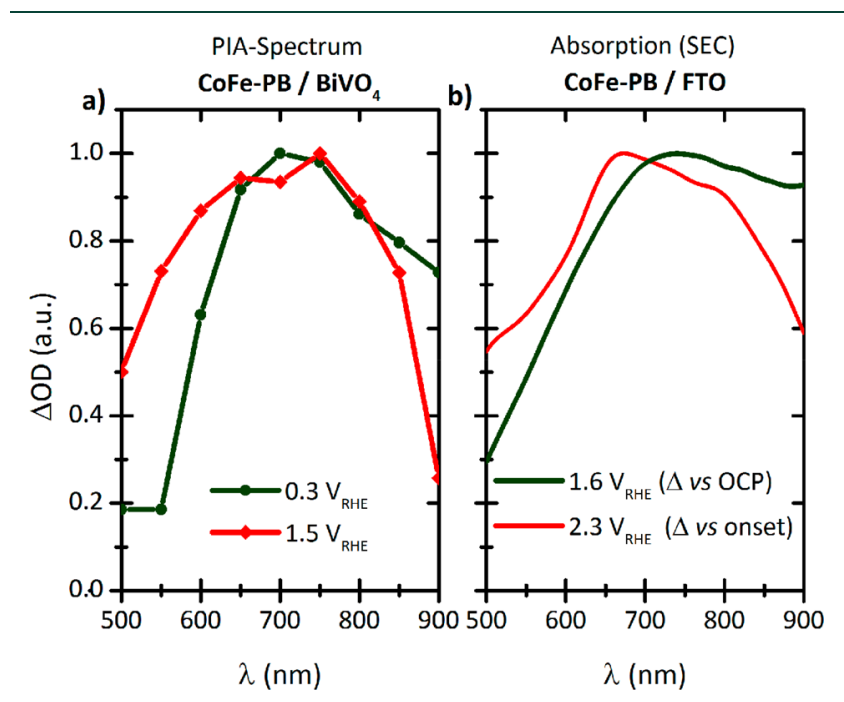

Figure 4. Normalized absorption spectra of CoFe-PB modified $\mathrm{BiVO}_{4}$ (a) obtained from PIAS and of solely CoFe-PB on FTO (b) obtained from spectroelectrochemistry (SEC) at low (below onset) bias (dark green) and high (above onset) bias (red).

CoFe-PB on FTO (Figures $4 \mathrm{~b}$ and $1 \mathrm{c}$ ), assigned to the subsequent generation of $\mathrm{CoFe}-\mathrm{PB}^{2+}$ states. These data thus indicate that under modest applied potential $\left(0.3 \mathrm{~V}_{\mathrm{RHE}}\right)$ hole transfer from $\mathrm{BiVO}_{4}$ to $\mathrm{CoFe}-\mathrm{PB}$ results primarily in the generation of unreactive (and therefore slowly decaying) $\mathrm{CoFe}-\mathrm{PB}^{+}$states. However, as the applied potential (and photocurrent) increases, irradiation results in the generation of $\mathrm{CoFe}-\mathrm{PB}^{2+}$ states capable of driving WO. The greater reactivity of these states is consistent with their faster PIA decay kinetics. Fitting the decay of PIA traces gives a time constant for WO by $\mathrm{CoFe}-\mathrm{PB}^{2+}$ states on the order of seconds (Figure S8b), in agreement with a previously reported time scale for electrocatalytic WO on CoFe-PB. ${ }^{19}$

In summary, and as illustrated in Scheme 1, our kinetic studies indicate that the enhanced photoelectrochemical WO performance of $\mathrm{CoFe}-\mathrm{PB}$ modified $\mathrm{BiVO}_{4}$ results from fast $(\mu \mathrm{s}$ or faster) hole transfer from $\mathrm{BiVO}_{4}$ to $\mathrm{CoFe}-\mathrm{PB}$. This charge transfer enables the generation of long-lived photogenerated holes (i.e $\mathrm{CoFe}-\mathrm{PB}^{+/ 2+}$ states in $\mathrm{CoFe}-\mathrm{PB}$ ) in the absence of strong anodic bias. WO kinetics are on the order of seconds on both $\mathrm{BiVO}_{4}$ and $\mathrm{CoFe}-\mathrm{PB}$, despite the more oxidizing nature of $\mathrm{BiVO}_{4}$ holes. The resultant suppression of recombination losses, without any compromise in WO kinetics, is the origin of the remarkable shift of the WO onset potential following CoFe-PB deposition.

The kinetics that we report herein for $\mathrm{CoFe}-\mathrm{PB}$ modified $\mathrm{BiVO}_{4}$ differ substantially from those that we have reported previously for $\mathrm{CoP}_{\mathrm{i}}$ modified dense $\mathrm{BiVO}_{4}$ films (Scheme $1 \mathrm{~b}$ ). $\mathrm{WO}$ on $\mathrm{CoP}_{\mathrm{i}}$ is $2-3$ orders of magnitude slower than WO via the $\mathrm{BiVO}_{4}$ surface or WO via CoFe-PB. This slow catalysis results in the accumulation of large amounts of relatively unreactive charge in $\mathrm{CoP}_{\mathrm{i}}$ when used as a co-catalyst, observed as slowly decaying PIA signals with amplitudes 2 orders of magnitude greater than those observed in CoFe-PB, where holes in the catalyst are removed from the catalyst by WO. ${ }^{14}$

However, these large signals arise from a $\mathrm{CoP}_{\mathrm{i}}$ layer 10 times thicker than the CoFe-PB layer studied herein (200 vs $20 \mathrm{~nm}$ ). Consequently, despite larger signals, only singly oxidized (i.e., precatalytic) $\mathrm{CoP}_{i}$ states are observed, corresponding to a lower hole density. This contrasts the multiply oxidized CoFePB states observed herein. This is consistent with the concept of enhanced performance following $\mathrm{CoP}_{\mathrm{i}}$ deposition resulting from retarded recombination within $\mathrm{BiVO}_{4}$ due to increased band bending, suggested to result from Schottky junction formation $^{10}$ (see Scheme 1b). A further difference is that in $\mathrm{CoP}_{\mathrm{i}} / \mathrm{BiVO}_{4}$ hole transfer to the $\mathrm{CoP}_{\mathrm{i}}$ was observed to be relatively slow and inefficient (occurring on order of seconds), despite similar apparent energy offsets for hole transfer (roughly $1 \mathrm{eV}$ ), and contributed little to the overall WO

Scheme 1. (a) Photon Conversion Efficiency in Unmodified $\mathrm{BiVO}_{4}$ Limited by the Kinetic Competition between PotentialDependent Surface Recombination ( $\mathrm{rec}(\mathrm{V})$ ) and WO, (b) CoP $\mathrm{P}_{\mathrm{i}}$ Modification Reducing the Applied Potential Needed to Effectively Suppress Surface Recombination, Allowing Holes to React via the $\mathrm{BiVO}_{4}$ surface, ${ }^{a}$ and (c) CoFe-PB Enhanceing the Efficiency via a Different Mechanism ${ }^{b}$

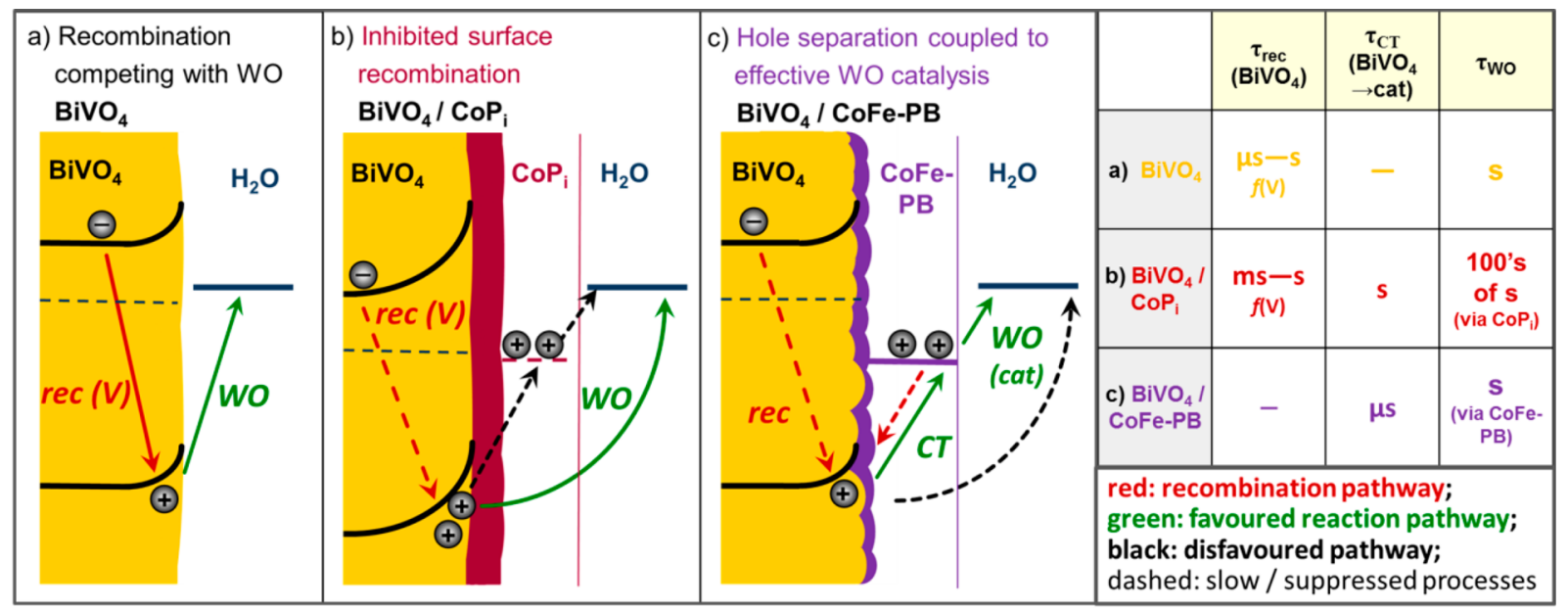

${ }^{a}$ Hole charge transfer $(\mathrm{CT})$ to $\mathrm{CoP}_{\mathrm{i}}$ and $\mathrm{WO}$ via $\mathrm{CoP}_{\mathrm{i}}$ is slow and does not contribute to photocurrent. ${ }^{b}$ Efficient hole CT to CoFe-PB separates holes from electrons in the $\mathrm{BiVO}_{4}$ surface (thereby suppressing this recombination pathway). This is coupled to effective WO catalysis. 
flux. $^{10,13,14}$ One possible explanation for this is that the interface between $\mathrm{CoFe}-\mathrm{PB}$ and the most thermodynamically favorable surface facets of $\mathrm{BiVO}_{4}$ is significantly more covalent (Figure S9) as it exhibits better epitaxy than $\mathrm{CoP}_{\mathrm{i}} / \mathrm{BiVO}_{4}$. Those covalent linkages can largely decrease the barrier for charge transfer from/to the nanostructured $\mathrm{BiVO}_{4}$ with high surface area, as studied herein. An alternative hypothesis is that this difference could be related to the difference in morphology between the $\mathrm{BiVO}_{4}$ studied as in our previous study of $\mathrm{CoP}_{\mathrm{i}}$ / $\mathrm{BiVO}_{4}$ a flat (dense) $\mathrm{BiVO}_{4}$ film with a planar junction to a thick $(200 \mathrm{~nm}) \mathrm{CoP}_{\mathrm{i}}$ layer was used. ${ }^{10}$ The $\mathrm{BiVO}_{4}$ samples studied herein are highly textured and interface via a high surface area junction to an extremely thin CoFe-PB layer (20 $\mathrm{nm})$. Research is currently underway to further investigate the conclusions of this work, in particular, the effect of the semiconductor and co-catalyst morphology as well as the semiconductor/co-catalyst interface on the efficiency of hole transfer as well as the factors that control the kinetics of WO, with the aim of constructing a set of simple and general design principles for assessing the compatibility of semiconductor/cocatalyst systems.

\section{ASSOCIATED CONTENT}

\section{S Supporting Information}

The Supporting Information is available free of charge on the ACS Publications website at DOI: 10.1021/acsenergylett.8b02225.

Material synthesis, description of the experimental setups, UV-vis spectroscopy and additional SEC data, TA spectra at different voltages, fitting of PIAS decay, crystalline models of $\mathrm{BiVO}_{4}$ and $\mathrm{CoFe}-\mathrm{PB}$ surfaces, and discussion of interface linkages (PDF)

\section{AUTHOR INFORMATION}

\section{Corresponding Authors}

*E-mail: 1.francas@ic.ac.uk (L.F.).

*E-mail: j.durrant@imperial.ac.uk (J.R.D.).

*E-mail: nlopez@iciq.es (N.L.).

*E-mail: jrgalan@iciq.es (J.-R.G.-M.).

\section{ORCID}

Sacha Corby: 0000-0001-7863-5426

Núria López: 0000-0001-9150-5941

Sixto Giménez: 0000-0002-4522-3174

José-Ramón Galán-Mascarós: 0000-0001-7983-9762

James Robert Durrant: 0000-0001-8353-7345

\section{Author Contributions}

\#B.M. and F.S.H. contributed equally to the manuscript. The manuscript was written through contributions of all authors. All authors have given approval to the final version of the manuscript.

\section{Notes}

The authors declare no competing financial interest.

\section{ACKNOWLEDGMENTS}

This work was funded by the European Union's Horizon 2020 project A-LEAF (Grant Agreement No. 732840); the Spanish Ministerio de Economía y Competitividad (MINECO) through Projects CTQ2015-71287-R, CTQ2015-68770-R, and ENE2017-85087-C3-1-R; the Generalitat de Catalunya (2017-SGR-1406 and 2014SGR-199); and the CERCA Programme/Generalitat de Catalunya. Additionally, the
"LaCaixa"-Severo Ochoa International Programme of Ph.D. Scholarships is acknowledged for F.S.H.'s predoctoral grant. The Engineering and Physical Sciences Research Council (EPSRC) is acknowledged for B.M.'s doctoral training studentship.

\section{ABBREVIATIONS}

$\mathrm{BiVO}_{4}=$ bismuth vanadate; $\mathrm{CoFe}-\mathrm{PB}=$ cobalt iron Prussian blue (cobalt hexacyanoferrate); $\mathrm{WO}=$ water oxidation; $\mathrm{SEC}=$ spectroelectrochemistry; TAS = transient absorption spectroscopy; PIAS = photoinduced absorption spectroscopy

\section{REFERENCES}

(1) Fujishima, A.; Zhang, X.; Tryk, T. A. $\mathrm{TiO}_{2}$ Photocatalysis and Related Surface Phenomena. Surf. Sci. Rep. 2008, 63, 515-582.

(2) Hardee, K. L.; Bard, A. J. Semiconductor Electrodes V. The Application of Chemically Vapor Deposited Iron Oxide Films to Photosensitized Electrolysis. J. Electrochem. Soc. 1976, 123, 10241026.

(3) Fàbrega, C.; Murcia-López, S.; Monllor-Satoca, D.; Prades, J. D.; Hernández-Alonso, M. D.; Penelas, G.; Morante, J.; Andreu, T. Efficient $\mathrm{WO}_{3}$ Photoanodes Fabricated by Pulsed Laser Deposition for Photoelectrochemical Water Splitting with High Faradaic Efficiency. Appl. Catal., B 2016, 189, 133-140.

(4) Park, J.; McDonald, K. J.; Choi, K.-S. Progress in Bismuth Vanadate Photoanodes for Use in Solar Water Oxidation. Chem. Soc. Rev. 2013, 42, 2321-2337.

(5) Peter, L. M.; Upul Wijayantha, U. K. Photoelectrochemical Water Splitting at Semiconductor Electrodes: Fundamental Problems and New Perspectives. ChemPhysChem 2014, 15, 1983-1995.

(6) Tamirat, A.; Rick, J.; Dubale, A.; Su, W.-N.; Hwang, B.-J. Using hematite for photoelectrochemical water splitting: a review of current progress and challenges. Nanoscale Horiz 2016, 1, 243-267.

(7) Steinmiller, E. M.; Choi, K.-S. Photochemical Deposition of Cobalt-Based Oxygen Evolving Catalyst on a Semiconductor Photoanode for Solar Oxygen Production. Proc. Natl. Acad. Sci. U. S. A. 2009, 106, 20633-20636.

(8) Zhong, D. K.; Gamelin, D. R. Photoelectrochemical Water Oxidation by Cobalt Catalyst ("Co-Pi") $/ \alpha-\mathrm{Fe}_{2} \mathrm{O}_{3}$ Composite Photoanodes: Oxygen Evolution and Resolution of a Kinetic Bottleneck. J. Am. Chem. Soc. 2010, 132, 4202-4207.

(9) Badia-Bou, L.; Mas-Marza, E.; Rodenas, P.; Barea, E. M.; Fabregat-Santiago, F.; Gimenez, S.; Peris, E.; Bisquert, J. Water Oxidation at Hematite Photoelectrodes with an Iridium-Based Catalyst. J. Phys. Chem. C 2013, 117, 3826-3833.

(10) Ma, Y.; Le Formal, F.; Kafizas, A.; Pendlebury, S. R.; Durrant, J. R. Efficient suppression of back electron/hole recombination in cobalt phosphate surface-modified undoped bismuth vanadate photoanodes. J. Mater. Chem. A 2015, 3, 20649-20657.

(11) Sinclair, T. S.; Gray, H. B.; Müller, A. M. Photoelectrochemical Performance of $\mathrm{BiVO}_{4}$ Photoanodes Integrated with (NiFe)-Layered Double Hydroxide Nanocatalysts. Eur. J. Inorg. Chem. 2018, 2018, 1060-1067.

(12) Kanan, M. W.; Nocera, D. G. In Situ Formation of an OxygenEvolving Catalyst in Neutral Water Containing Phosphate and $\mathrm{Co}^{2+}$. Science 2008, 321, 1072-1075.

(13) Zachäus, C.; Abdi, F. F.; Peter, L. M.; van de Krol, R. Photocurrent of $\mathrm{BiVO}_{4}$ is Limited by Surface Recombination, not Surface Satalysis. Chem. Sci. 2017, 8, 3712-3719.

(14) Ma, Y.; Kafizas, A.; Pendlebury, S. R.; Le Formal, F.; Durrant, J. R. Photoinduced Absorption Spectroscopy of $\mathrm{CoPi}$ on $\mathrm{BiVO}_{4}$ : The Function of CoPi during Water Oxidation. Adv. Funct. Mater. 2016, 26, 4951-4960.

(15) Ma, Y.; Mesa, C. A.; Pastor, E.; Kafizas, A.; Francàs, L.; Le Formal, F.; Pendlebury, S. R.; Durrant, J. R. Rate Law Analysis of Water Oxidation and Hole Scavenging on a BiVO4 Photoanode. ACS Energy Lett. 2016, 1, 618-623. 
(16) Ma, Y.; Pendlebury, S. R.; Reynal, A.; Le Formal, F.; Durrant, J. R. Dynamics of Photogenerated Holes in Undoped $\mathrm{BiVO}_{4}$ Photoanodes for Solar Water Oxidation. Chem. Sci. 2014, 5, 2964-2973.

(17) Surendranath, Y.; Kanan, M. W.; Nocera, D. G. Mechanistic Studies of the Oxygen Evolution Reaction by a Cobalt-Phosphate Catalyst at Neutral pH. J. Am. Chem. Soc. 2010, 132, 16501-16509.

(18) Hegner, F. S.; Herraiz-Cardona, I.; Cardenas-Morcoso, D.; Lopez, N.; Galan-Mascaros, J.-R.; Gimenez, S. Cobalt Hexacyanoferrate on $\mathrm{BiVO}_{4}$ Photoanodes for Robust Water Splitting. ACS Appl. Mater. Interfaces 2017, 9, 37671-37681.

(19) Pintado, S.; Goberna-Ferrón, S.; Escudero-Adán, E. C.; GalánMascarós, J.-R. Fast and Persistent Electrocatalytic Water Oxidation by Co-Fe Prussian Blue Coordination Polymers. J. Am. Chem. Soc. 2013, 135, 13270-13273.

(20) Goberna-Ferrón, S.; Hernández, W. Y.; Rodríguez-García, B.; Galán-Mascarós, J.-R. Light-Driven Water Oxidation with Metal Hexacyanometallate Heterogeneous Catalysts. ACS Catal. 2014, 4, $1637-1641$.

(21) Indra, A.; Paik, U.; Song, T. Boosting Electrochemical Water Oxidation with Metal Hydroxide Carbonate Templated Prussian Blue Analogues. Angew. Chem., Int. Ed. 2018, 57, 1241-1245.

(22) Han, L.; Tang, P.; Reyes-Carmona, A.; Rodriguez-Garcia, B.; Torrens, M.; Morante, J.; Arbiol, J.; Galan-Mascaros, J.-R. Enhanced Activity and Acid pH Stability of Prussian Blue-type Oxygen Evolution Electrocatalysts Processed by Chemical Etching. J. Am. Chem. Soc. 2016, 138, 16037-16045.

(23) Hegner, F. S.; Cardenas-Morcoso, D.; Gimenez, S.; Lopez, N.; Galan-Mascaros, J.-R. Level Alignment as Descriptor for Semiconductor/Catalyst Systems in Water Splitting: The Case of Hematite/Cobalt Hexacyanoferrate Photoanodes. ChemSusChem 2017, 10 (10), 4552-4560.

(24) Lezna, R. O.; Romagnoli, R.; de Tacconi, N. R.; Rajeshwar, K. Cobalt Hexacyanoferrate: Compound Stoichiometry, Infrared Spectroelectrochemistry, and Photoinduced Electron Transfer. J. Phys. Chem. B 2002, 106, 3612-3621.

(25) Kulesza, P. J.; Zamponi, S.; Malik, M. A.; Berrettoni, M.; Wolkiewicz, A.; Marassi, R. Spectroelectrochemical Characterization of Cobalt Hexacyanoferrate Films in Potassium Salt Electrolyte. Electrochim. Acta 1998, 43, 919-923.

(26) Pastor, E.; Le Formal, F.; Mayer, M. T.; Tilley, D. S.; Francàs, L.; Mesa, C. A.; Grätzel, M.; Durrant, J. R. Spectroelectrochemical Analysis of the Mechanism of (Photo)Electrochemical Hydrogen Evolution at a Catalytic Interface. Nat. Commun. 2017, 8, 14280. 